\title{
Soft Stenosis of the Lumbar Spine: Thickness vs Hypertrophy of the Ligamentum Flavum. A Pathogenetic and Molecular Point of View
}

Alessandro Landi* and Roberto Delfini

Department of Neurology and Psychiatry, Division of Neurosurgery, University of Rome Sapienza, Italy

\begin{abstract}
In the literature, there is no longer agreement neither on the real existence neither on the pathogenic mechanism that causes ligamentous lumbar stenosis or "soft stenosis". In particular, the main questions are: 1 - is it caused by the hypertrophy of the ligamentum flavum or by its withdrawal into the spinal canal due to the loss of elasticity and the disc collapse? 2 - is there a molecular substrate that can explain the hypertrophy of the ligamentum flavum? Lately, the identification of the fractalkine's overexpression demonstrated a fundamental role of the metameric instability and of the joint inflammation in the pathogenesis of hypertrophy of the ligamentum flavum, thus making clear the association between joint hypermobility and soft spinal stenosis.
\end{abstract}

The study of this association is worthy of more clinical and instrumental findings, even if recent studies have shown growing evidence that the soft stenosis is a clinic-pathological well-defined entity. Its primum movens seems to be the vertebral instability and its molecular substrate seem to be the overexpression of fractalkine, going to place in the unstable phase of the degenerative cascade of the lumbar spine.

Keywords: LF hypertrophy; LF thickness; Soft stenosis; Hard stenosis; Lumbar spine; Fractalkine

\section{Manuscript}

In the literature, there is no longer agreement neither on the real existence neither on the pathogenic mechanism that causes ligamentous lumbar stenosis or "soft stenosis". In particular, the main questions are: 1 - is it caused by the hypertrophy of the ligamentum flavum or by its withdrawal into the spinal canal due to the loss of elasticity and the disc collapse? 2 - is there a molecular substrate that can explain the hypertrophy of the ligamentum flavum? [1-4]. The purpose is to make clearer the pathogenetic mechanism responsible for the most common degenerative spinal pathology.

The ligamentum flavum, or yellow ligament, is so called because of the macroscopically slightly yellow color due to the high concentration of elastin and collagen fibers within its structure, in particular elastin makes up about $60-70 \%$ of the extracellular matrix. From an anatomical point of view the yellow ligament plays a fundamental role in metameric biomechanics because it is a significant component of the lateral and posterior wall of the spinal canal and, from a biomechanical point of view, has two fundamental characteristics: elasticity, given by the high concentration of elastin, and rigidity, conferred by collagen fibers. The combination of these two features makes the yellow ligament extremely elastic and resistant to mechanical stresses. Age and inflammatory processes cause a reduction in the concentration of elastin in the yellow ligament, with consequent changes of the collagen/ elastin ratio in favor of the second one $[3,5,6]$. These processes generate calcification, ossification and chondrometaplasia of the ligament, which gradually loses elasticity and acquires stiffness, going in hypertrophy and increasing in volume [3]. The loss of elasticity, associated with disc degeneration and loss of height of the intervertebral space (primum movens of the activation of the lumbar degenerative cascade) causes protrusion of the ligament in the spinal canal going to reduce the diameter of it. All these processes represent the pathogenetic substrate on which lumbar stenosis develops. Anatomo-pathological studies have demonstrated how the reversal of the collagen/elastin ratio age-related is evident only on the dorsal layer of the yellow ligament, whereas the anterior layer in contact with the dura mater does not present such alteration $[1-3,5,6]$. This happens because the mechanical stress generated by the hypermobility of the metamere, mainly acts on the dorsal layer of the ligament which then undergoes a greater number of micro-traumas: the repair of such microtraumas produces an increase of the collagen fibers in the dorsal layer which degenerates and goes in hypertrophy. So the presence of the LF hypertrophy is proved and well described in literature.

But what is the molecular substrate of the hypertrophy? Several hypotheses have been proposed to validate the presence of a molecular substrate that causes the yellow ligament hypertrophy and consequent soft stenosis. In my opinion the most reliable theories are 3:

Overexpression of collagen type I mRNA: Numerous studies have shown that age causes overexpression of the collagen type I mRNA in the LF and that this is favored by the fact that mechanical stress increases the expression of TGFbeta mRNA, which stimulates the expression of collagen type I. Therefore it is evident that age and mechanical stress on the metamere cause the increasing production of collagen in the LF $[1,2,7,8]$. So a predominant role in the pathogenesis of soft stenosis is due to the metameric hypermobility; greater is the metameric motility, greater is the hypertrophic response of the yellow ligament. This, however, is not enough to explain why the yellow ligament becomes hypertrophied and increases considerably in volume at the expense of the spinal canal.

Increased expression of matrix metalloproteinases: These molecules are enzymes that degrade all kinds of extracellular matrix components such as elastin, collagen and proteoglycans and are normally responsible for the remodeling of the connective tissue in physiological and pathological conditions. In particular there is an overexpression of this group of enzymes in inflammatory and rheumatic

*Corresponding author: Alessandro Landi, Department of Neurology and Psychiatry, Division of Neurosurgery, University of Rome Sapienza, Viale de Policlinico 155, 00181, Rome, Italy, Tel: +390649979105; Fax: +390649979105 E-mail: dott.alessandro.landi@gmail.com

Received November 12, 2013; Accepted November 14, 2013; Published November 17, 2013

Citation: Landi A, Delfini R (2013) Soft Stenosis of the Lumbar Spine: Thickness vs Hypertrophy of the Ligamentum Flavum. A Pathogenetic and Molecular Point of View. J Spine 2: e111. doi:10.4172/2165-7939.1000e111

Copyright: @ 2013 Landi A, et al. This is an open-access article distributed under the terms of the Creative Commons Attribution License, which permits unrestricted use, distribution, and reproduction in any medium, provided the original author and source are credited. 
diseases, including herniated disc and vertebral instability. They are classified into three subtypes. In particular MMP2 is responsible for the degradation of elastin. This subtype of metalloproteinases has been isolated in high concentrations in the yellow ligament of the patients with lumbar stenosis [9]. This, however, is not enough to explain what is the pathogenetic mechanism that causes increased expression of MMP2 in LF.

Overexpression of Fractalkine: Lately the overexpression a chemokine, the fractalkine, has been found in the hypertrophic yellow ligament. In particular, it is well documented its role in the development of numerous inflammatory diseases (rheumatoid arthritis, dermatitis, etc.) and in ligaments and joints involved in inflammatory processes caused by instability (e.g., joint capsules, ligaments, and synovium). The inflammatory process involves these tissues so the fractalkine overexpression is activated; thus causing the recruitment of mononuclear cells within the LF feeding the inflammation and causing vascular injury and angiogenesis [10-15]. Moreover such an increase in mononuclear activity cause a proliferation of fibroblasts, (for overexpression of TGF beta mRNA resulting in increased collagen fibers) and inflammatory cells in LF. This inflammatory cells activity in the LF causes rupture of the extracellular matrix (for activation of metalloproteinase MMP2) due to the elastin degradation, resulting in loss of elasticity of the ligament and subsequent hypertrophy. This seems to be a clear explanation of why the thickening of the yellow ligament is due to an increase in inflammatory cells and to an acceleration on the mechanism of damage - repair of the LF. This explains both the increase of volume and the inversion of the elastin/collagen ratio which is evident in patients with soft stenosis.

\section{Conclusions}

In my opinion the answer to the main questions about the real existence of the soft stenosis, does not lie in the attempt to exclude one or the other theory, but rather in a consequentiality of them. In particular, the overexpression of fractalkine, make us understand the fundamental role of the metameric instability and of the articular inflammation in the pathogenesis of the yellow ligament hypertrophy, thus making clear the association between joint hypermobility and soft spinal stenosis. Moreover, its overexpression makes clear the association between it and the consequential activation of the mRNA expression of TGF beta and metalloproteinases MMP2, highlighting how these processes are not isolated but part of a degenerative cascade activated by the hypermobility. The study of this association is worthy of more clinical and instrumental findings, even if recent studies show growing evidence that the soft stenosis is a clinic-pathological well-defined entity. Its primum movens seems to be the vertebral instability and its molecular substrate seem to be the overexpression of fractalkina, going to place in the unstable phase of the degenerative cascade of the spine.

\section{References}

1. Honsawek S, Poonpukdee J, Chalermpanpipat C, Payungporn S, Limthongku $W$, et al. (2013) Hypertrophy of the ligamentum flavum in lumbar spinal canal stenosis is associated with increased bFGF expression. Int Orthop 37: 13871392.
2. Zhong ZM, Zha DS, Xiao WD, Wu SH, Wu Q, et al. (2011) Hypertrophy of ligamentum flavum in lumbar spine stenosis associated with the increased expression of connective tissue growth factor. J Orthop Res 29: 1592-1597.

3. Kosaka H, Sairyo K, Biyani A, Leaman D, Yeasting R, et al. (2007) Pathomechanism of loss of elasticity and hypertrophy of lumbar ligamentum flavum in elderly patients with lumbar spinal canal stenosis. Spine ( $\mathrm{Phila} \mathrm{Pa}$ 1976) 32: 2805-2811.

4. Altinkaya N, Yildirim T, Demir S, Alkan O, Sarica FB (2011) Factors associated with the thickness of the ligamentum flavum: is ligamentum flavum thickening due to hypertrophy or buckling? Spine (Phila Pa 1976) 36: E1093-1097.

5. Lakemeier S, Schmid R, Foltz L, Rohlfs J, Fuchs-Winkelmann S, et al. (2012) Increased expression of CD44 in hypertrophied ligamentum flavum and relevance of splice variants CD44v5 and CD44v6. Acta Neurochir 154: 359 365

6. Sairyo K, Biyani A, Goel V, Leaman D, Booth Jr R, et al. (2005) Pathomechanism of Ligamentum Flavum Hypertrophy: A Multidisciplinary Investigation Based on Clinical, Biomechanical, Histologic, and Biologic Assessments. Spine 30: 26492656.

7. Kim HJ, Park JB, Won HY, Chang H (2007) Serum Levels of TGF-beta1, TIMP1 and TIMP-2 in Patients with Lumbar Spinal Stenosis and Disc Herniation. Asian Spine J 1: 8-11.

8. Chen YT, Wei JD, Wang JP, Lee HH, Chiang ER, et al. (2011) Isolation of mesenchymal stem cells from human ligamentum flavum: implicating etiology of ligamentum flavum hypertrophy. Spine (Phila Pa 1976) 36: E1193-1200.

9. Park JB, Kong CG, Suhl KH, Chang ED, Riew KD (2009) The increased expression of matrix metalloproteinases associated with elastin degradation and fibrosis of the ligamentum flavum in patients with lumbar spinal stenosis. Clin Orthop Surg 1: 81-89.

10. Shah R, Hinkle CC, Ferguson JF, Mehta NN, Li M, et al. (2011) Fractalkine is a novel human adipochemokine associated with type 2 diabetes. Diabetes 60 $1512-1518$.

11. Ruth JH, Volin MV, Haines GK 3rd, Woodruff DC, Katschke KJ Jr, et al. (2001) Fractalkine, a novel chemokine in rheumatoid arthritis and in rat adjuvantinduced arthritis. Arthritis Rheum 44: 1568-1581.

12. Sunnemark D, Eltayeb S, Nilsson M, Wallström $E$, Lassmann $H$, et al. (2005) CX3CL1 (fractalkine) and CX3CR1 expression in myelin oligodendrocyte glycoprotein-induced experimental autoimmune encephalomyelitis: kinetics and cellular origin. J Neuroinflammation 2: 17.

13. Bjerkeli V, Damås JK, Fevang B, Holter JC, Aukrust P, et al. (2007) Increased expression of fractalkine (CX3CL1) and its receptor, CX3CR1, in Wegener's granulomatosis--possible role in vascular inflammation. Rheumatology (Oxford) 46: $1422-1427$.

14. Davis CN, Zujovic V, Harrison JK (2004) Viral macrophage inflammatory protein-II and fractalkine (CX3CL1) chimeras identify molecular determinants of affinity, efficacy, and selectivity at CX3CR1. Mol Pharmacol 66: 1431-1439.

15. Yang XP, Mattagajasingh S, Su S, Chen G, Cai Z, et al. (2007) Fractalkine Upregulates Intercellular Adhesion Molecule-1 in Endothelial Cells Through CX3CR1 and the Jak - Stat5 Pathway Circ Res 101: 1001-1008. 